\title{
A minimizing principle for the Poisson-Boltzmann equation
}

\author{
A.C. Maggs \\ Laboratoire PCT, Gulliver CNRS-ESPCI UMR 7083, 10 rue Vauquelin, 75231 Paris Cedex 05.
}

\begin{abstract}
The Poisson-Boltzmann equation is often presented via a variational formulation based on the electrostatic potential. However, the functional has the defect of being non-convex. It can not be used as a local minimization principle while coupled to other dynamic degrees of freedom. We formulate a convex dual functional which is numerically equivalent at its minimum and which is more suited to local optimization.
\end{abstract}

\section{Introduction}

The Poisson-Boltzmann treatment of free ions is widely used in simulations with implicit solvents. It successfully describes the mean field screening properties of ionic solutions. At the most phenomenological level it is found by balancing two crucial features of the ionic system: The electrostatic energy coming from Coulomb's law, plus the ideal entropy of mixing of the ions. Written in terms of ion concentrations $c_{j}$, or the charge concentrations $\rho_{j}=c_{j} q_{j}$ we find the free energy of a solution from

$$
\begin{array}{r}
F=\int d^{3} \mathbf{r} d^{3} \mathbf{r}^{\prime} \rho(\mathbf{r}) \frac{1}{4 \pi \epsilon_{0}\left|\mathbf{r}-\mathbf{r}^{\prime}\right|} \rho\left(\mathbf{r}^{\prime}\right)+ \\
k_{B} T \int d^{3} \mathbf{r} \sum_{j}\left(c_{j} \ln \left(c_{j} / c_{j 0}\right)-c_{j}\right)
\end{array}
$$

where the total charge density $\rho=\sum_{j} \rho_{j}+\rho_{f} . \rho_{f}$ is an external fixed charge density - associated with surfaces or molecular sources, $\boldsymbol{c}_{\mathbf{j} 0}$ is the reference density of component $j$. If we minimize this functional of $c_{j}$ then we find an effective free energy for the source field $\rho_{f}$, [1]. The problem in this formulation is the appearance of the long-ranged Coulomb interaction [2] which renders the evaluation and minimization less efficient than might be wished.

We start with a few points of notation: We will use the symbol $f$ to describe a density of free energy of an electrolyte in mean field theory, $h$ denotes a complex action which occurs in a functional integral and $u$ is the electrostatic energy of the electric field. We will freely integrate by parts in our expressions dropping boundary terms. The transformations that we perform conserve the stationary point of the mean field solution which is denoted $f_{s}$. The statistical weight of a configuration is then

$$
w=e^{-\beta \int f_{s} d^{3} \mathbf{r}}
$$

no matter the arguments of the functional $f$. $\rho_{f}$ is kept constant during all variations of the electric and ionic degrees of freedom. We denote the Legendre transform of a convex function $g(x)$ as

$$
\tilde{g}(\xi)=\mathcal{L}(g(x))[\xi]=\sup _{x}(x \xi-g(x))
$$

In eq. (1) one conventionally decouples the electrostatic interactions by introducing the potential as an additional variational field. If we do so then we find that

$$
f=\rho \phi-\epsilon \frac{(\nabla \phi)^{2}}{2}+\sum_{j} k_{B} T\left(c_{j} \ln \left(c_{j} / c_{j 0}\right)-c_{j}\right)
$$

We see at once that we have gained in locality of the formulation; the other advantage is that it is valid for arbitrary spatial variation in the dielectric properties, $\epsilon(\mathbf{r})$. Unfortunately, the counterpart is that the resulting free energy is no-longer convex. We do not have a minimizing principle, rather a stationary principle. This excludes some of the simplest optimization strategies that one might want apply - such as simultaneous annealing of conformational and electrostatic degrees of freedom. From eq. (4) one studies the differential

$$
d f=\sum_{j} d c_{j}\left(q_{j} \phi+k_{B} T \ln \left(c_{j} / c_{j 0}\right)\right)+d \phi(\rho+\nabla \cdot \epsilon \nabla \phi)
$$

We now impose that the coefficient of $d_{c}$ is zero so that

$$
\mathrm{q}_{j} \phi+\mathrm{k}_{\mathrm{B}} T \ln \left(\mathrm{c}_{\mathrm{j}} / \mathrm{c}_{\mathrm{j} 0}\right)=0
$$

Substituting back into eq. (4) we find the standard form for the Poisson-Boltzmann functional [3-7]:

$$
f=\rho_{f} \phi-\epsilon \frac{(\nabla \phi)^{2}}{2}-k_{B} T \sum_{j} c_{j 0} e^{-q_{j} \phi \beta}
$$

We take the variations of this functional with respect to $\phi$ to find:

$$
\rho_{f}+\nabla \cdot \epsilon \nabla \phi+\sum_{j} q_{j} c_{j 0} e^{-\beta q_{j} \phi}=0
$$

For the symmetric ion system

$$
\begin{array}{r}
f=\rho_{f} \phi-\epsilon \frac{(\nabla \phi)^{2}}{2}-2 k_{B} T c_{0} \cosh (\beta q \phi) \\
\rho_{f}+\nabla \cdot \epsilon \nabla \phi-2 q c_{0} \sinh (\beta q \phi)=0
\end{array}
$$

Eq. (8) continues to be awkward numerically since clearly both the derivative and the cosh functions are unbounded below; simple annealing procedures are thus unstable. One has to solve the partial differential equation eq. (9) in practical applications $[8,9]$.

The purpose of this paper is to derive functionals that are equivalent to those given above which combine the 
advantages of the convexity of eq. (1) and the locality of eq. (4). We show how to find functions which are numerically equivalent to those widely used in the literature. We consider this absolutely crucial since much time has already been invested in calibration of potential functions. We thus look for ways of rendering convex known, accurate functionals. Our main tool in this effort will be the Legendre transform in a form presented in [10] as a reciprocal principle for variational calculations. In the following calculations the way that the energy $\epsilon(\nabla \phi)^{2} / 2$ is transformed into an equivalent form $\mathbf{D}^{2} / 2 \epsilon$ will remind the reader of the transformation of the kinetic energy in a Lagrangian, $m \dot{x}^{2} / 2$ into the Hamiltonian equivalent, $p^{2} / 2 m$. This is a standard example of the use of Legendre transforms in classical mechanics.

We start by summarizing the state of the art in the calculation of effective free energies for Poisson-Boltzmann free energies, and then showing in a simple example how the ideas of dynamically constrained fields can be used to write a convex free energy in terms of the electric displacement field $\mathbf{D}$. We then generalize the procedure to arbitrary formulations of the free energy demonstrating the link to the theory of Legendre transforms.

\section{Field theory route to Poisson-Boltzmann}

In recent years theoretical methods have been introduced to generalize the application of Poisson-Boltzmann equations to a larger range of systems, [11, 12]. All these methods are based on the Hubbard-Stratonovich transformation to break pair interactions into one-body potentials. They have shown their power in producing functionals that include the finite volume of ions as well as mobile dipoles. The Hubbard-Stratonovich transformation produces a complex action, which can however be studied at its saddle point. In this case one reproduces the known Poisson-Boltzmann functionals for simple situations, and in addition study fluctuations about the mean field solution in terms of a loop expansion.

A typical example of such a function for both symmetric ions and dipoles is [13-15]

$$
\begin{aligned}
& f=\rho_{f} \phi-\epsilon_{0} \frac{(\nabla \phi)^{2}}{2}- \\
& \frac{k_{B} T}{a^{3}} \ln \left(1+2 \lambda_{\text {ion }} \cosh (\beta q \phi)+\lambda_{d i p} \frac{\sinh \left(\beta p_{0}|\nabla \phi|\right)}{\beta p_{0}|\nabla \phi|}\right)
\end{aligned}
$$

where $\lambda_{\text {ion }}$ and $\lambda_{\text {dip }}$ are the chemical potentials of the ions and the dipoles, $a$ a hard core size, $p_{0}$ a dipole moment. $\epsilon(\mathbf{r})=\epsilon_{0}$, since dielectric effects are generated dynamically. The logarithmic term in the energy is found from studying the partition function of a lattice gas.

\section{Constrained fields plus mixing entropy}

An alternative, local approach to electrostatics was introduced in [16-18] to avoid the use of Ewald and fast Fourier methods in electrostatic codes. The ideas were applied to the Poisson-Boltzmann equation in [19, 20]. A convex energy is associated with the Gauss law constraint which is implemented dynamically. In particular we reproduce the mean field Poisson-Boltzmann equations from the functional

$$
\begin{gathered}
f=\frac{\mathbf{D}^{2}}{2 \epsilon}+k_{B} T \sum_{j}\left(c_{j} \ln \left(c_{j} / c_{j 0}\right)-c_{j}\right) \\
\text { with } \operatorname{div} \mathbf{D}-\rho=0
\end{gathered}
$$

In the case of a one-component plasma one trivially eliminates the density degree of freedom to find

$$
\begin{aligned}
& f=\frac{D^{2}}{2 \epsilon}+k_{B} T s\left(\left(\operatorname{div} D-\rho_{f}\right) / q\right) \\
& s(z)=z \ln \left(z / c_{0}\right)-z
\end{aligned}
$$

The functional eq. (13) is now an unconstrained functional of the field $\mathbf{D}$. It can be discretized as in [16] where the vector field $\mathbf{D}$ is associated to the links of a cubic lattice, while scalar quantities such as (div D) and $\rho_{\mathrm{f}}$ are associated with the vertexes of the lattice. Eq. (13) has the advantage of being both convex and local.

\section{Reciprocity and Legendre transforms}

We now show how to transform a Poisson-Boltzmann functionals expressed in terms of electrostatic potentials $\phi$ into those based on the electric fields, D. Start with the electrostatic energy density

$$
u=\rho_{\mathrm{f}} \phi-\epsilon \frac{(\nabla \phi)^{2}}{2}
$$

As is well known the Poisson equation is found as the stationary condition of eq. (15). Introduce now $\mathbf{E}=-\nabla \phi$ using a Lagrange multiplier $\mathbf{D}$ to find the constrained functional

$$
u=\rho_{f} \phi-\epsilon \frac{\mathbf{E}^{2}}{2}+\mathbf{D} \cdot(\mathbf{E}+\nabla \phi)
$$

Integrate by parts and regroup:

$$
u=-\epsilon \frac{\mathbf{E}^{2}}{2}+\mathbf{D} \cdot \mathbf{E}-\phi\left(\operatorname{div} \mathbf{D}-\rho_{\mathrm{f}}\right)
$$

We now eliminate $\mathbf{E}$ and see the equivalence to

$$
u=\frac{\mathbf{D}^{2}}{2 \epsilon}-\phi\left(\operatorname{div} \mathbf{D}-\rho_{\mathrm{f}}\right)
$$

We now re-interpret the field $\phi$ as a Lagrange multiplier [10] imposing Gauss' law and find the starting point of 
our previous papers [16] on constrained statistical mechanics in electrostatics.

Let us now generalize this approach to PoissonBoltzmann functionals expressed in terms of the potential.

$$
f=\rho_{f} \phi-\epsilon \frac{(\nabla \phi)^{2}}{2}-g(\phi)
$$

We will explicitly treat the example of the symmetric electrolyte solution where $g(\phi)=2 k_{B} T c_{0} \cosh (\beta q \phi)$. The transformation of eqs. (15-18) still goes through and we find

$$
f=\frac{\mathbf{D}^{2}}{2 \epsilon}+\phi\left(\rho_{f}-\operatorname{div} \mathbf{D}\right)-g(\phi) .
$$

Further variations with respect to $\phi$ give simply the Legendre transform, eq. (3), of the function $g(\phi)$, where the transform variable is $\xi=\left(\rho_{f}-\operatorname{div} \mathbf{D}\right)$

To continue the transformation we require the stationary point of

$$
\phi \xi-2 k_{B} T c_{0} \cosh (\beta q \phi)
$$

finding $\phi=\left(k_{B} T / q\right) \sinh ^{-1}\left(\xi / 2 q c_{0}\right)$ and

$$
\mathcal{L}(\mathrm{g})[\xi]=\frac{k_{\mathrm{B}} T \xi}{\mathrm{q}} \sinh ^{-1}\left(\xi / 2 q c_{0}\right)-k_{\mathrm{B}} T \sqrt{4 c_{0}^{2}+\xi^{2} / \mathrm{q}^{2}}
$$

For symmetric solutions we conclude that the reciprocal functional that we require is

$$
f=\frac{\mathbf{D}^{2}}{2 \epsilon}+\mathcal{L}(\mathrm{g})\left[\rho_{\mathrm{f}}-\operatorname{div} \mathbf{D}\right]
$$

This demonstrates the principle result of the present paper. Starting from a standard, concave functional expressed in terms of the potential we have found a convex functional of the vector field $\mathbf{D}$. The critical points of the two functionals are, however, numerically identical.

When there are no ions within a region - for instance within a macromolecule - the Legendre transform requires some care in its definition: We take $g(\phi)=\eta \phi^{2} / 2$ with $\eta$ small. Then the Legendre transform is $\tilde{g}=$ $\xi^{2} /(2 \eta)$. Using finite $\eta$ gives a Yukawa or Debye interaction, with screening of the electrostatics. The limit $\eta \rightarrow 0$ imposes a delta-function constraint on Gauss' law. Experience with local Monte Carlo algorithms based on the electric field $[16,17]$ implies that relaxation of longitudinal and transverse degrees of freedom via link, and plaquette updates is the most efficient manner to sample the above functionals.

\section{Dipolar systems}

We now consider the transformation of the functional eq. (10) for small chemical potentials $\lambda_{j}$, where we expand the logarithm to lowest order. In this case we find a contribution of the form

$$
\mathbf{f}_{\mathbf{E}}=-\epsilon_{0} \frac{\mathbf{E}^{2}}{2}-\bar{\lambda}_{\mathrm{d}} \frac{\sinh \left(\beta p_{\mathrm{o}}|\mathbf{E}|\right)}{\beta p_{\mathrm{o}}|\mathbf{E}|}+\mathbf{D} \cdot \mathbf{E}
$$

Again if we consider the extremal equation for $\mathbf{E}$ we recognize the Legendre transform, this time for the electric field, rather than for the potential. We have here defined $\bar{\lambda}_{j}=k_{B} T \lambda_{j} / a^{3}$.

Thus the fully transformed functional in presence of both ions and dipoles is

$$
\begin{aligned}
f=\mathcal{L}\left(\epsilon_{0} \frac{\mathbf{E}^{2}}{2}+\right. & \left.\bar{\lambda}_{\text {dip }} \frac{\sinh \left(\beta p_{o}|\mathbf{E}|\right)}{\beta p_{0}|\mathbf{E}|}\right)[\mathbf{D}]+ \\
& 2 \bar{\lambda}_{\text {ion }} \mathcal{L}(\cosh (\beta e \phi))\left[\operatorname{div} \mathbf{D}-\rho_{\mathrm{f}}\right]
\end{aligned}
$$

For small fields, E, we expand the first line of eq. (25) and find $\epsilon_{0} \frac{\mathbf{E}^{2}}{2}\left(1+\bar{\lambda}_{\mathrm{dip}} \beta^{2} p_{0}^{2} / 3 \epsilon_{0}\right)$. The modification of the curvature of the function is a manifestation of the electric susceptibility the dipoles.

\section{Evaluating transforms}

In general it is impossible to analytically transform the functions needed in the dual or reciprocal formulation. In particular the full functional eq. (10) is not a simple sum of terms as assumed in the derivation of eq. (25). However in a given ionic system the Legendre transformed functionals are uniform in space. They should be calculated just once for a given set of chemical potentials. For the general problem we require the two-dimensional transform with respect to the variables $(\phi, \mathbf{E})$. Standard, fast numerical methods [21] are available for performing these transforms, and the result can be tabulated for a given application. For the highest accuracy an iterative Newton-Raphson step can be used to converge the interpolated results to very high accuracy. Forces can be evaluated as well as energies, for instance in eq. (22), $\mathrm{d} \tilde{\mathrm{g}}(\xi) / \mathrm{d} \xi=\phi-$ a general property of the transform [22], thus the force on a test particle with charge $e_{j}$ is just

$$
\mathbf{F}_{j}=-e_{j} \nabla \phi=e_{j} \mathbf{E}
$$

\section{Link with Infimal Convolution}

The Legendre transformation of the entropy function $s(z)$ of eq. (14) is the Boltzmann factor

$$
\mathcal{L}(\mathrm{s}(\boldsymbol{z}))[\xi]=\mathrm{c}_{0} \mathrm{e}^{\xi}
$$

appearing in eq. (6). The fact that the effective action eq. (6) is a sum of exponential functions shows that the reciprocal action must be linked to infimal convolution of the original free energy defined as follows [23]:

$$
\mathcal{L}(\tilde{g}+\tilde{d})[x]=\inf _{y}[g(y)+d(x-y)]
$$


We now show why this is the case for the symmetric electrolyte.

Consider a symmetric electrolyte with unit charges, $q=1$ and with reference concentrations $c_{0}=1$. We impose Gauss' law with a Lagrange multiplier $\phi$ and study the constrained minimum of the total ionic entropy

$$
t\left(c_{1}, c_{2}\right)=s\left(c_{1}\right)+s\left(c_{2}\right)+\phi\left(\xi-c_{1}+c_{2}\right)
$$

where $\xi=\left(\operatorname{div} \mathbf{D}-\rho_{\mathrm{f}}\right)$. The variation with respect to $\boldsymbol{c}_{j}$ gives

$$
\tilde{\mathrm{t}}(\phi)=-\tilde{\mathrm{s}}(-\phi)-\tilde{\mathrm{s}}(\phi)+\phi \xi
$$

We now study this Legendre transform of the sum of two functions. For the symmetric electrolyte we require (from the above definition of the infimal convolution)

$$
t^{\prime}(\xi)=\inf _{y}[y \ln (y)-y+(y-\xi) \ln (y-\xi)-(y-\xi)]
$$

giving $y(y-\xi)=1, y=\left(\xi+\sqrt{4+\xi^{2}}\right) / 2$. Thus the action for the symmetric electrolyte eq. (22) is found as the infimal convolution of two ideal entropy functions with the help of the identity $\sinh ^{-1}(\xi / 2)=\ln \left(\left(\xi+\sqrt{4+\xi^{2}}\right) / 2\right)$

\section{Fluctuations and Fourier transforms}

We now return to the full field theoretical formulation of the Poisson-Boltzmann equation after HubbardStratonovich transformation of the action, now denoted $h$, but before the saddle point evaluation [11, 12]. As a concrete example we consider the symmetric electrolyte:

$$
\begin{aligned}
h & =\epsilon \frac{(\nabla \phi)^{2}}{2}-2 k_{B} T c_{0} \cos (\beta q \phi)-i \rho_{f} \phi \\
& =\epsilon \frac{(\nabla \phi)^{2}}{2}+g(\phi)-i \rho_{f} \phi
\end{aligned}
$$

We now consider transformation in the philosophy of our above reciprocal formulation, but replacing Lagrange multipliers by complex integral representations of the delta-function. We do not neglect fluctuations in the fields and do not make any approximation in the statistical mechanics of the ionic system. Following very closely the logic described above we find the following succession of transformations of the action:

$$
\begin{aligned}
h & =\epsilon \frac{(\nabla \phi)^{2}}{2}+g(\phi)-i \rho_{f} \phi \\
& \rightarrow \epsilon \frac{\mathbf{E}^{2}}{2}+g(\phi)-i \mathbf{D} \cdot(\nabla \phi+\mathbf{E})-i \rho_{\mathrm{f}} \phi \\
& \rightarrow \epsilon \frac{\mathbf{E}^{2}}{2}+g(\phi)-i \mathbf{D} \cdot \mathbf{E}+i \phi\left(\operatorname{div} \mathbf{D}-\rho_{\mathrm{f}}\right) \\
& \rightarrow \frac{\mathbf{D}^{2}}{2 \epsilon}+g(\phi)+i \phi\left(\operatorname{div} \mathbf{D}-\rho_{\mathrm{f}}\right)
\end{aligned}
$$

The only thing that remains is the integral over $\phi$. This we recognize as a Fourier transform with variable $\xi=$ $\left(\operatorname{div} \mathbf{D}-\rho_{\mathrm{f}}\right)$. Thus the action expressed in terms of $\mathbf{D}$ is

$$
\mathrm{h}=\frac{\mathbf{D}^{2}}{2 \epsilon}-\ln \left\{\mathcal{F}\left(\mathrm{e}^{-\mathrm{g}(\phi)}\right)\right\}\left[\operatorname{div} \mathbf{D}-\rho_{\mathrm{f}}\right]
$$

with $\mathcal{F}$ the Fourier transform.

This action should be integrated over to find the partition sum

$$
\mathrm{Z}=\int \mathrm{d} \mathbf{D} e^{-\beta \int h \mathrm{~d}^{3} \mathbf{r}}
$$

When the fluctuations at the saddle point are neglected the Fourier transform reduces to the Legendre transform as above, and the infimal convolution is a simple echo of the standard convolution of statistical weights occurring at the saddle point. If the Fourier transform becomes negative then the action becomes complex, or one must at least sample functions which are non-positive. Again in general we do not expect to be able to evaluate the Fourier transform analytically but again tabulation for a specific problem is possible.

\section{Conclusions}

To conclude, we have introduced a duality transformation for Poisson-Boltzmann functionals which allows us to find a local minimizing principle for both electrostatic and conformation degrees of freedom in a simulation. This opens the perspective of simpler annealing and dynamic relaxation in molecular simulation, including local Car-Parrinello evolution of ionic degrees of freedom [17] for complex molecules. Clearly the interpolation of source charges to a grid requires control of their self-energy in a manner which in familiar in molecular dynamics simulations [24-26].

The functionals are designed to exactly conserve the saddle point. This is only true in practice if the numerical discretization is strictly equivalent. The final formulation requires a discretized divergence for Gauss' law in eq.(20), "Div". This divergence operator is adjoint to the "-Grad" from eq. (16). Finally the Laplacian in the potential formulation must obey $\nabla^{2}=$ Div Grad. If this is not true discretization errors differ between the formulations.

The original and reciprocal functionals have very different forms- as noted above a weakly constraining energy $\eta \phi^{2} / 2$, with $\eta$ small, is transformed into a steep constraint $\xi^{2} /(2 \eta)$, and vice-versa. In the future it will be interesting to understand how this influences mathematical descriptions of fluctuations about the mean field behaviour. As stated in [10] the potential and field formulations can be used together to give upper and lower bounds for the mean field free energy. 
[1] Jianwei Che, Joachim Dzubiella, Bo Li, and J. Andrew McCammon, "Electrostatic free energy and its variations in implicit solvent models," The Journal of Physical Chemistry B 112, 3058-3069 (2008), pMID: 18275182, http://pubs.acs.org/doi/pdf/10.1021/jp7101012.

[2] Hartmut Löwen, Paul A. Madden, and Jean-Pierre Hansen, " $A b$ initio description of counterion screening in colloidal suspensions," Phys. Rev. Lett. 68, 1081-1084 (1992).

[3] Federico Fogolari and James M. Briggs, "On the variational approach to poisson-boltzmann free energies," Chemical Physics Letters 281, 135 - 139 (1997).

[4] Kim A. Sharp and Barry. Honig, "Calculating total electrostatic energies with the nonlinear poisson-boltzmann equation," The Journal of Physical Chemistry 94, 7684-7692 (1990), http://pubs.acs.org/doi/pdf/10.1021/j100382a068.

[5] Wonpil Im, Dmitrii Beglov, and Benoit Roux, "Continuum solvation model: Computation of electrostatic forces from numerical solutions to the poisson-boltzmann equation," Computer Physics Communications 111, 59 - 75 (1998).

[6] Eric S. Reiner and Clayton J. Radke, "Variational approach to the electrostatic free energy in charged colloidal suspensions: general theory for open systems," Journal of the Chemical Society, Faraday Transactions 86, 39013912 (1990).

[7] Michael K. Gilson, Malcolm E. Davis, Brock A. Luty, and J. Andrew McCammon, "Computation of electrostatic forces on solvated molecules using the poisson-boltzmann equation," The Journal of Physical Chemistry 97, 3591-3600 (1993), http://pubs.acs.org/doi/pdf/10.1021/j100116a025.

[8] Ray Luo, Laurent David, and Michael K. Gilson, "Accelerated poisson-boltzmann calculations for static and dynamic systems," Journal of Computational Chemistry 23, 1244-1253 (2002).

[9] B Honig and A Nicholls, "Classical electrostatics in biology and chemistry," Science 268, 1144-1149 (1995), http://www.sciencemag.org/content/268/5214/1144.full.pdf.

[10] R. Courant and D. Hilbert, Methods of Mathematical Physics: Volume I, Chapter 4.9 (John Wiley and Sons, 1989).

[11] R. R. Netz and H. Orland, "Field theory for charged fluids and colloids," EPL (Europhysics Letters) 45, 726732 (1999).

[12] R.R. Netz and H. Orland, "Beyond poisson-boltzmann:
Fluctuation effects and correlation functions," The European Physical Journal E: Soft Matter and Biological Physics 1, 203-214 (2000), 10.1007/s101890050023.

[13] Ariel Abrashkin, David Andelman, and Henri Orland, "Dipolar poisson-boltzmann equation: Ions and dipoles close to charge interfaces," Phys. Rev. Lett. 99, 077801 (2007).

[14] I. Borukhov, D. Andelman, and H. Orland, "Adsorption of large ions from an electrolyte solution: a modified poisson-boltzmann equation," Electrochimica Acta 46, $221-229$ (2000).

[15] Cyril Azuara, Henri Orland, Michael Bon, Patrice Koehl, and Marc Delarue, "Incorporating dipolar solvents with variable density in poisson-boltzmann electrostatics," Biophysical journal 95, 5587-5605 (2008).

[16] A. C. Maggs and V. Rossetto, "Local simulation algorithms for coulomb interactions," Phys. Rev. Lett. 88, 196402 (2002).

[17] Jörg Rottler and A. C. Maggs, "Local molecular dynamics with coulombic interactions," Phys. Rev. Lett. 93, 170201 (2004).

[18] A. C. Maggs and R. Everaers, "Simulating nanoscale dielectric response," Phys. Rev. Lett. 96, 230603 (2006).

[19] A. C. Maggs, "Auxiliary field monte carlo for charged particles," The Journal of Chemical Physics 120, 31083118 (2004).

[20] M. Baptista, R. Schmitz, and B. Dünweg, "Simple and robust solver for the poisson-boltzmann equation," Phys. Rev. E 80, 016705 (2009).

[21] Yves Lucet, "Faster than the fast legendre transform, the linear-time legendre transform," Numerical Algorithms 16, 171-185 (1997), 10.1023/A:1019191114493.

[22] R. K. P. Zia, Edward F. Redish, and Susan R. McKay, "Making sense of the Legendre transform," American Journal of Physics 77, 614-622 (2009).

[23] R. T. Rockafellar, Convex Analysis (Princeton Mathematical Series) (Princeton Univ Pr, 1970).

[24] M. Deserno and C. Holm, "How to mesh up ewald sums. i. a theoretical and numerical comparison of various particle mesh routines," J. Chem. Phys. 109, 7678 (1998).

[25] Ulrich Essmann, Lalith Perera, Max L. Berkowitz, Tom Darden, Hsing Lee, and Lee G. Pedersen, "A smooth particle mesh ewald method," J. Chem. Phys. 103, 85778593 (1995).

[26] Yibing Shan, John L. Klepeis, Michael P. Eastwood, Ron O. Dror, and David E. Shaw, "Gaussian split ewald: A fast ewald mesh method for molecular simulation," J. Chem. Phys. 122, 054101 (2005). 